\title{
Mobile miRNAs for stem cell maintenance
}

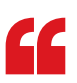

\section{a mobile}

miR394 signal

emanating

from the

protogerm

represses $L C R$

in the stem cell region
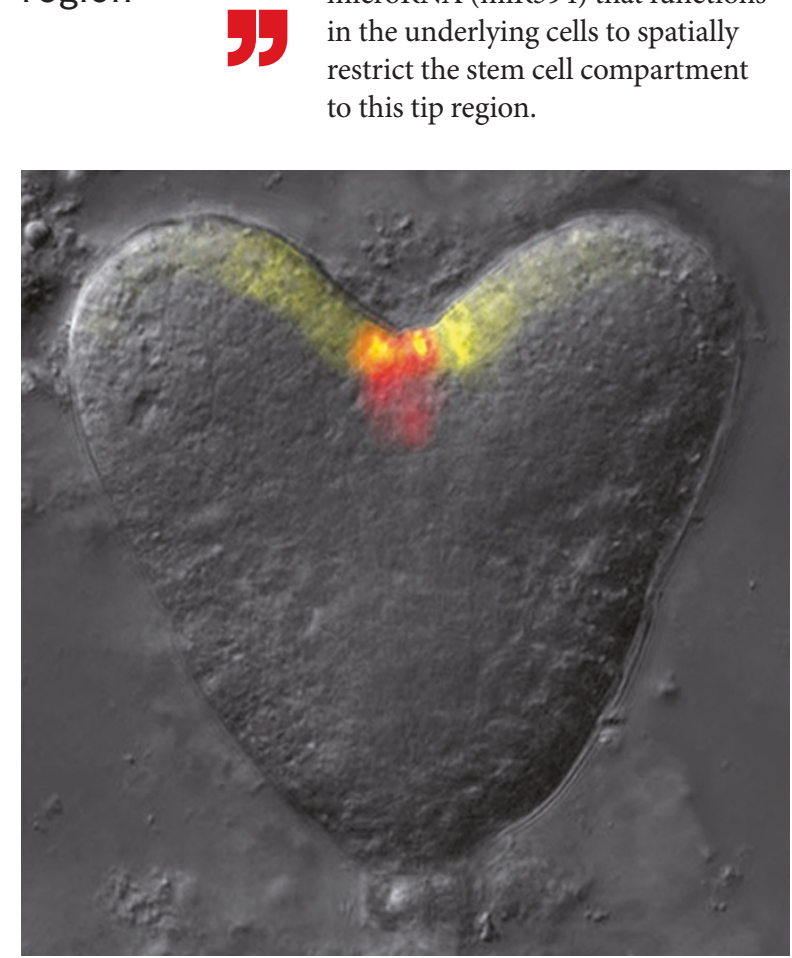

An Arabidopis thaliana embryo at the stage when the stem cell layers (red) have just been established in the shoot meristem region between the two outgrowing cotyledonary primordia. The protoderm cells that provide the micro RNA miR394 signal are marked in yellow. Image courtesy of T. Laux, Albert-Ludwigs-University Freiburg, Germany.
All aerial organs of a plant originate from the shoot apical meristem (SAM), which contains a population of pluripotent stem cells at the tip. The fate of these stem cells is dictated by their position in the SAM, but the underlying molecular basis of this spatial control is unclear. Laux and colleagues now show that the surface cell layer L1 (also known as the protoderm) is the source of a microRNA (miR394) that functions in the underlying cells to spatially restrict the stem cell compartment to this tip region.
The gene encoding miR394 was cloned in a screen for Arabidopsis thaliana mutants that enhanced a weak argonaute 10 (ago10-1) stem cell mutant in which meristems terminate rarely. Enhancer mutations promoted the loss of stem cell activity from the SAM, increasing meristem termination and growth arrest. Plants lacking miR394 expression still initiated stem cell programmes during early embryogenesis and expressed the stem cell regulator WUSCHEL (WUS) but were unable to maintain stem cells despite WUS expression.

Among the known predicted targets of miR394, Laux and colleagues found that downregulation of the F-box protein LCR (LEAF CURLING RESPONSIVENESS) was required for stem cell function. Indeed, expression of miR394resistant $L C R$ mRNA caused meristem termination, and this could be rescued by expressing a mutated version of miR394 that restored targeting of this resistant LCR mRNA.

To further investigate how miR394 regulates stem cells, the authors determined where it is expressed. They found that the MIR394 gene was ubiquitously expressed during early embryogenesis and then became restricted to the $\mathrm{L} 1$ of the embryo shoot apex. However, mature miR394 was not restricted to the L1 and instead formed a graded signal that extended to the underlying cell layers (L2 and L3). This suggested that miR394 might function as a mobile signal to maintain all three stem cell layers.

Interestingly, the authors found that miR394-mediated silencing occurs throughout the apical cell layers of the embryo. However, region-specific analysis showed that miR394-mediated silencing of $L C R$ activities is only required in the stem cell niche in order to maintain stem cell function, whereas it has no effect in the cells surrounding the stem cell region.

Together these results indicate that a mobile miR394 signal emanating from the protoderm represses $L C R$ in the stem cell region and is essential for stem cell maintenance. Thus, the protoderm seems to provide a spatial cue by linking stem cell competence to the distal layers of the SAM. As LCR promotes protein degradation via the proteasome, the authors argue that LCR repression at the tip of the SAM prevents the degradation of signalling components that act downstream of WUS and thus allows stem cell pluripotency to be maintained.

Kim Baumann

ORIGINAL RESEARCH PAPER Knauer, S. et al. A protodermal miR394 signal defines a region of stem cell competence in the Arabidopsis shoot meristem. Dev. Cell 17 Jan 2013 (http://dx.doi. org/10.1016/j.devcel.2012.12.009) 